\title{
Do bone mineral content and density determine fracture in children? A possible threshold for physical activity
}

\author{
Ana Martins ${ }^{1}$, Teresa Monjardino $^{1}$, Luísa Nogueira ${ }^{2}$, Helena Canhão ${ }^{3}$ and Raquel Lucas ${ }^{1}$
}

BACKGROUND: Relations between bone parameters, physical exertion, and childhood fractures are complex. We aimed to estimate the associations between fracture history and bone mineral content $(\mathrm{BMC})$ and areal bone mineral density (aBMD) at 7 years of age, by levels of physical activity, as a proxy for trauma frequency.

METHODS: We used data collected from 2,261 children of the Generation XXI birth cohort, assembled in 2005/6 in Porto, Portugal. At the age of 7 years (2012/4), fracture history, time spent per week in active play, and sports practice were reported by parents. Subtotal and lumbar spine (LS) BMC and aBMD were measured using whole-body dual-energy X-ray absorptiometry.

RESULTS: Boys and girls in the highest categories of time spent in sports practice or active play generally had higher $\mathrm{BMC}$ and $\mathrm{aBMD}$. Among girls, $\mathrm{BMC}$ and $\mathrm{aBMD}$ were protective of fracture only in the highest quarter of active play (>660 min/week) —odds ratios (OR; 95\% confidence interval $(95 \% \mathrm{Cl}))$ for subtotal $\mathrm{BMC}=0.27(0.11-0.67)$, subtotal $\mathrm{aBMD}=$ $0.18(0.06-0.49)$, and $L S$ aBMD $=0.41(0.22-0.75)$. For boys in the highest quarter of sports practice ( $>240 \mathrm{~min} /$ week), subtotal and LS BMC were protective of fracture- $-O R=0.39$ (0.16-0.98) and $0.51(0.27-0.96)$, respectively.

CONCLUSION: In prepubertal children, BMC and $\mathrm{aBMD}$ predicted fracture history only in the highest levels of physical activity.

C hildhood fractures are common and account for $10-25 \%$ of all pediatric injuries (1). The accumulated risk of sustaining a fracture from birth until 16 years of age was estimated as $27 \%$ for girls and $42 \%$ for boys (2). Fracture incidence peaks at $\sim 10-12$ years in girls and $13-15$ years in boys, which coincides with the growth spurt $(1,3,4)$. The most common anatomical site of fracture in children is the upper limb, mainly the forearm (5). Fractures have short-term impact on children's lives as they may result in the time off from school and activity-restricted days. In the long term, fractures can also lead to relevant degenerative changes, such as secondary osteoarthritis (6).
Childhood fracture can also be seen as an early marker of bone fragility as children who experience fractures have, on average, decreased bone mass $(7,8)$. In a life-course perspective (9), skeletal deficits during childhood may also track significantly throughout life $(10,11)$, and empirical research suggests that children who sustain a fracture are more likely to have decreased bone mass during adulthood $(12,13)$, which is well known to increase the risk of fragility fracture in older ages (14).

During childhood, the contribution of parameters such as bone mineral content (BMC) or areal bone mineral density (aBMD) to fracture risk is particularly difficult to assess in non-athletes, as pediatric fractures frequently result from moderate- to high-energy trauma, which can hardly be attributed to underlying bone deficits alone $(6,15,16)$. To make it even more challenging, it is accepted that common levels of physical activity have a dual effect on fracture. On the one hand, physical exertion contributes directly to an increase in fracture risk due to higher inherent exposure to injury (e.g., falls and collisions) (17). On the other hand, it has a protective effect on the risk of fracture due to a mechanical stimulus of bone formation, which potentially improves overall bone strength (18).

This set of mechanisms can be summarized in the following premises: (i) densitometric bone properties such as aBMD and BMC are inversely associated with fracture risk (19); (ii) intense physical activity is directly and independently associated with fracture risk due to increased trauma probability (18); and (iii) intense physical activity has a positive effect on BMC and aBMD (18-22). Taken together, these findings may seem contradictory; however, a possible explanation may be an interaction of bone parameters with physical activity to produce fracture. Specifically, it is plausible that the relation between $\mathrm{aBMD} / \mathrm{BMC}$ and fracture risk is modified by trauma frequency and severity, i.e., the protective role of $\mathrm{aBMD} / \mathrm{BMC}$ on fracture may vary with the level of physical exertion imposed, regardless of the osteogenic contribution of the latter to bone formation. Therefore, to explore the extent to which fractures in the pediatric population reflect the burden of lifelong bone fragility, the relative contribution of $\mathrm{aBMD} / \mathrm{BMC}$ should be estimated

${ }^{1}$ EPIUnit-Instituto de Saúde Pública, Universidade do Porto, Porto, Portugal; ${ }^{2}$ Department of Radiology, School of Health Technology of Porto/Polytechnic Institute of Porto (ESTSP/IPP), Porto, Portugal; ${ }^{3}$ EpiDoC, CEDOC, Nova Medical School, NOVA University, Lisbon, Portugal. Correspondence: Raquel Lucas (rlucas@med.up.pt)

Received 30 November 2016; accepted 15 April 2017; advance online publication 24 May 2017. doi:10.1038/pr.2017.113 
according to the regular level of exposure to trauma in a biological interaction perspective. Trauma frequency is challenging to assess; however, there is evidence that both organized sports and leisure-time activity are clear predictors of injury risk (17) and can be used as their proxy. To our knowledge, no study so far has investigated this mechanistic interaction hypothesis.

Thus, in the present study, we aimed to estimate the associations of fracture history with BMC and aBMD, by levels of physical activity, in a large population-based sample of 7-year-old children.

\section{METHODS}

\section{Participants}

This study was conducted within the Generation XXI (G21) population-based birth cohort. Briefly, the cohort was assembled during 2005-2006 in five public maternity units, covering six municipalities of the metropolitan area of Porto, Portugal. Mothers who delivered a live-born (gestational age $\geqslant 24$ weeks) were invited to participate in the cohort in $24-72 \mathrm{~h}$ following delivery. At this stage, $91.4 \%$ of the invited mothers accepted to participate, which resulted in 8,647 infants being included in the cohort (23). Between April 2012 and April 2014, 5,849 children (79.6\% of the initial sample) were successfully evaluated in person in the 7-year-old follow-up wave. Among those, all children consecutively evaluated between 1 December 2012 and 31 August $2013(n=3,015)$ were additionally invited to undergo a whole-body dual-energy X-ray absorptiometry (DXA) scan. Of those, 2,421 (80.3\%) underwent the scan and the remaining either refused, or missed, the scheduled exam three or more times. After excluding 13 children with invalid scans due to movement, artifacts, or other logistic issues, 139 children with missing information on physical activity, and 8 with no data on fracture history, our final sample for the present study was composed of 2,261 children.

\section{Ethics Statement}

The Generation XXI study was approved by the University of Porto Medical School/São João Hospital Centre ethics committee and by the National Committee for Data Protection. Written informed consent according to the Helsinki Declaration was obtained from parents or legal guardians in all evaluations.

\section{Data Collection Procedures}

Outcome assessment. Children's lifetime history of fracture, the anatomical site, and number of fractures, as well as the age at the first fracture at each site, were assessed through a face-to-face structured questionnaire applied to parents. Parental report of fracture has shown acceptable validity in a different setting (24). In a small subsample of the G21 cohort $(n=74)$, the reproducibility of fracture report by parents within 1 year was $95 \%$. For analysis, all fractures sustained prior to 2 years of age $(n=32)$ were excluded as these are most probably due to severe trauma unrelated to bone quality or child behavior, such as delivery injuries, child abuse, or accidental falls due to caregiver's negligence $(25,26)$.

Table 1. Anthropometric and behavioral characteristics, body composition, and fracture history at 7 years of age according to sex

\begin{tabular}{|c|c|c|c|}
\hline & Girls $(n=1,066)$ & Boys $(n=1,195)$ & $P$ \\
\hline Mean age (SD; years) & $7.4(0.4)$ & $7.4(0.4)$ & $0.202^{\mathrm{a}}$ \\
\hline Mean weight (SD; kg) & $27.3(5.9)$ & $27.2(5.2)$ & $0.632^{\mathrm{a}}$ \\
\hline Mean BMI (SD; $\left.\mathrm{kg} / \mathrm{m}^{2}\right)$ & $17.6(2.8)$ & $17.2(2.4)$ & $0.001^{\mathrm{a}}$ \\
\hline Mean subtotal fat mass $(\mathrm{SD} ; \mathrm{kg})$ & $8.5(3.6)$ & $7.0(3.1)$ & $<0.001^{\mathrm{a}}$ \\
\hline Mean subtotal BMC (SD; g) & $591.2(85.9)$ & $601.1(85.6)$ & $0.006^{\mathrm{a}}$ \\
\hline Mean subtotal aBMD (SD; $\left.\mathrm{g} / \mathrm{cm}^{2}\right)$ & $0.612(0.057)$ & $0.624(0.053)$ & $<0.001^{a}$ \\
\hline Mean lumbar spine BMC (SD; g) & $18.6(3.5)$ & $18.8(3.7)$ & $0.182^{\mathrm{a}}$ \\
\hline Mean lumbar spine aBMD (SD; $\left.\mathrm{g} / \mathrm{cm}^{2}\right)$ & $0.684(0.067)$ & $0.669(0.063)$ & $<0.001^{\mathrm{a}}$ \\
\hline Yes & $56(5.2 \%)$ & $65(5.4 \%)$ & \\
\hline
\end{tabular}

Time spent in sports practice per week (min)

$0.017^{c}$

50th percentile (25th; 75 th)

$135(90 ; 225)$

$165(90 ; 240)$

Time spent in active plays per week (min)

$0.026^{\mathrm{C}}$

50th percentile (25th; 75 th)

$480(270 ; 660)$

$510(270 ; 720)$

$\mathrm{aBMD}$, areal bone mineral density; $\mathrm{BMC}$, bone mineral content

Data are presented as mean and SD, counts ( $n$ ), and frequencies (\%) or as median and percentiles (25th percentile, 75 th percentile). $P$-values under 0.05 are presented in bold. astudent's t-test.

${ }^{b} x^{2}$-text.

'Mann-Whitney U-test. 


\section{Anthropometrics and Body Composition Assessment}

Anthropometric measures at 7 years of age were obtained by trained interviewers while children stood barefoot in light indoor clothing. Height $(\mathrm{cm})$ and weight $(\mathrm{kg})$ were measured to the nearest tenth using a wall stadiometer (Seca, Hamburg, Germany) and a digital scale (Tanita Arlington Heights, IL, USA), respectively. BMI $\left(\mathrm{kg} / \mathrm{m}^{2}\right)$ was calculated through the ratio of weight to squared height.

DXA was performed using a Hologic Discovery QDR 4,500 W device (Hologic Inc., Bedford, MA, USA), and the following bone parameters were determined: $\mathrm{BMC}(\mathrm{g})$ and $\mathrm{aBMD}\left(\mathrm{g} / \mathrm{cm}^{2}\right)$, measured for the subtotal-whole body minus the head-and lumbar spine (LS) regions. Additionally, subtotal lean mass (kg), fat mass ( $\mathrm{kg})$, and total fat percentage were also measured during the scan. Bone parameters and body composition $z$-scores were calculated separately for each sex, as the difference between each participant's parameter and the sample mean, divided by the sample SD.

\section{Physical Activity Assessment}

Physical activity was measured using a standardized questionnaire that comprised a quantitative assessment of the average time spent by the child in different activities during weekdays and weekends over the previous year-sleeping, sitting, using the computer, engaging in active play, and practicing organized sports. The questionnaire, like most existing tools used to quantify physical activity in children, was developed to estimate energy expenditure specifically in terms of metabolic equivalents of task. However, in this particular work, our aim was to use physical activity measures as proxies for trauma frequency rather than to estimate energy expenditure. In the absence of a commonly accepted method to assess trauma frequency in prepubertal children, we opted to classify participants in terms of time spent in moderate-to-vigorous activities per week. Specifically, average time spent per day in activities such as physically demanding play, riding a bicycle, running, or other types of active play was recorded for weekdays and weekends, and time spent in active play per week was calculated. Time spent in organized sports activities per week was recorded using the same method.

As we found significant differences in the distribution of time spent in the practice of sports and active play between boys and girls (Table 1), we estimated sex-specific quartiles of each variable. For girls, the resulting quarters of time spent in sports practice were $[0$, 90], [91, 135], [136, 225], and [226, 990] $\mathrm{min} / \mathrm{week}$, and for active play, the quarters were $[0,270],[271,480],[481,660]$, and $[661$, $2,700] \mathrm{min} /$ week. In boys, the quarters were $[0,90],[91,165],[166$, $240]$, and $[241,675] \mathrm{min} /$ week for time spent in sports practice, and $[0,270],[271,510],[511,720]$, and $[721,2160] \mathrm{min} /$ week for time spent in active play.

\section{Statistical Analysis}

We restricted the statistical analysis to children who had complete information on fracture history, physical activity, and aBMD/BMC, and no weighting technique or missing data imputation was conducted. To assess the differences between children included and excluded from the sample as well as between boys and girls included, anthropometric characteristics and age were compared using Student's $t$-test, whereas physical activity was compared using Mann-Whitney's test and fracture history using the $\chi^{2}$-test.Because of the well-documented sexual dimorphism observed since early stages, also supported by our findings of significant differences between boys and girls in bone and anthropometric parameters as well as fracture sites and physical activity, all analyses were stratified by sex. This option was biologically rather than empirically grounded, as none of the statistical terms for sex $\times$ BMC and sex $\times a B M D$ interactions in regression models with the fracture history as dependent variable were statistically significant, which could be expected, given the absence of a clear association between $\mathrm{BMC} / \mathrm{aBMD}$ and fracture.

The frequency of fracture history was compared between categories of time spent in sports practice and active play using $\chi^{2}$-tests for linear trend. The mean BMC and aBMD trends across categories of time spent in sports practice and active play were estimated using linear regressions adjusted for height, weight, and age. The associations of $\mathrm{aBMD} / \mathrm{BMC}$ and body composition with fracture history (one or more fractures up to the age of 7 vs. no fracture) were estimated using odds ratios (ORs) and 95\% confidence intervals (95\% CIs) calculated using logistic regression. Crude and adjusted for height, weight, and age OR estimates are presented.

We intended to explore the potential modifying role of physical activity on the relation between $\mathrm{BMC} / \mathrm{aBMD}$ and fracture, in a biological interaction perspective. Therefore, our analytical approach was to stratify the analysis a priori by physical activity level (categories of time spent in active play and in sports practice) in order to assess whether ORs between BMC/aBMD and fracture history were different in magnitude by levels of physical activity, suggesting different biological mechanisms. Empirically, the significance of regression coefficients found for the interaction between LS BMC and the highest categories of sports practice $(P=0.036)$, and between LS BMD and active play $(P=0.057)$, suggested that the possibility of interaction should not be excluded, even though the remaining interaction terms tested had nonsignificant coefficients.

Statistical analysis was performed using Stata version 11.2 for Windows (Stata, College Station, TX).

\section{RESULTS}

\section{Study Sample}

Children included in the analysis $(n=2,261)$ were slightly lighter $(25.9$ vs. $26.4 \mathrm{~kg}, \quad P<0.001)$, shorter $(123.4$ vs. $123.8 \mathrm{~cm}, P=0.001)$, and had a marginally lower BMI (16.9 vs. $\left.17.1 \mathrm{~kg} / \mathrm{m}^{2}, P<0.001\right)$ than those in the remaining cohort. Children included were also slightly younger (7.1 vs. 7.2 years, $P<0.001)$ and reported more time spent in sports practice (median $\left(25^{\text {th }}\right.$ percentile, $75^{\text {th }}$ percentile $\left.\left(\mathrm{P}_{25}, \mathrm{P}_{75}\right)\right)-150$ (90, $240)$ vs. $120(60,180) \mathrm{min} /$ week, $P<0.001)$ but less time spent in active play $(510(270,690)$ vs. $510(290,780) \mathrm{min} /$ week, $P=0.002)$. There were no differences in the prevalence of fracture between children included in the analysis and the remaining cohort $(5.4 \%$ vs. $5.4 \%, P=0.194)$.

\section{Fracture Occurrence}

In the sample, $121(5.4 \%)$ children sustained at least one fracture and a total of 141 fractures were reported. The number of fractures generally increased with age and was higher in boys than in girls. In addition, almost $60 \%$ of all fractures occurred after the age of 4 years (Supplementary Figure S1). The majority of fractures occurred in the upper limb (>50\%), both in boys and in girls throughout all ages. Girls presented a higher proportion of lower limb fractures than boys at each age (15-30\% vs. 5-16\%; Supplementary Figure S2).

\section{Anthropometry, Body Composition, Physical Activity, and Fracture History}

At 7 years of age, boys were significantly taller than girls, but girls had higher BMI. Boys had higher subtotal BMC (601.1 vs. $591.2 \mathrm{~g}, P=0.006)$ and $\operatorname{aBMD}\left(0.624\right.$ vs. $0.612 \mathrm{~g} / \mathrm{cm}^{2}$, $P<0.001$; Table 1). However, girls presented higher LS aBMD than boys $\left(0.684\right.$ vs. $\left.0.669 \mathrm{~g} / \mathrm{cm}^{2}, P<0.001\right)$. Girls also had higher fat mass ( 8.5 vs. $7.0 \mathrm{~kg}, P<0.001)$ and fat percentage (35.3\% vs. $29.5 \%, P<0.001)$, whereas boys had higher lean mass (15.8 vs. $14.7 \mathrm{~kg}, P<0.001)$. Physical activity levels were 

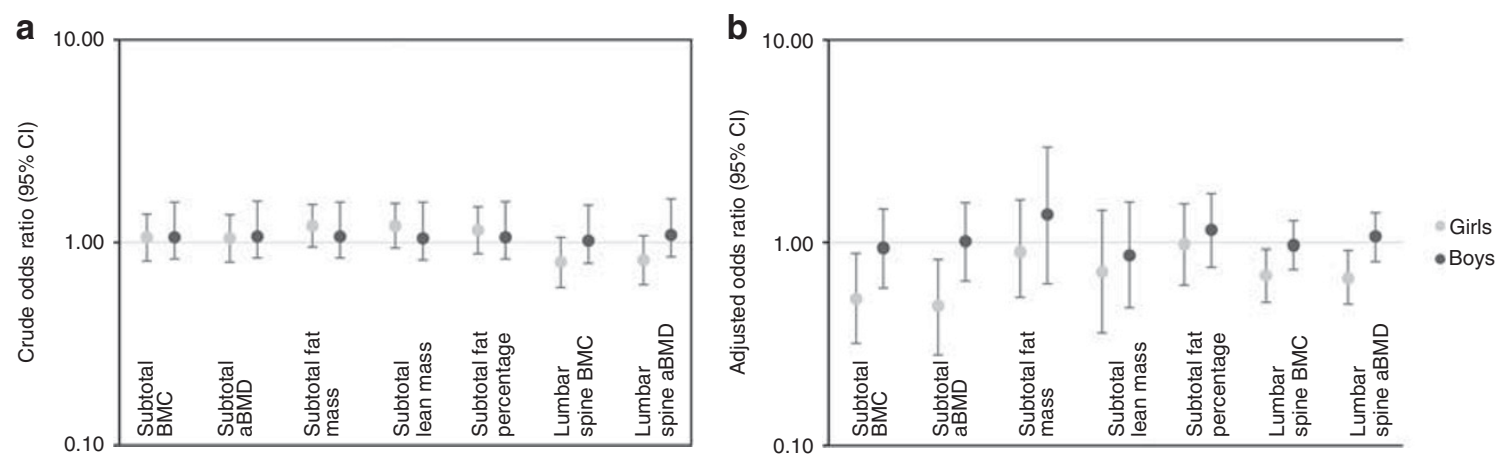

Figure 1. Odds ratios and $95 \%$ confidence intervals for the associations between body composition parameters and fracture history (any fracture vs. no fracture) according to sex. (a) Crude; (b) adjusted for height, weight, and age. aBMD, areal bone mineral density; BMC, bone mineral content; $95 \%$ $\mathrm{Cl}, 95 \%$ confidence interval. The number of children with fracture history was 56 out of 1,055 girls and 65 out of 1,178 boys.

higher in boys, who spent more time than girls in active play - median $\left(\mathrm{P}_{25}, \mathrm{P}_{75}\right): 510(270,720)$ vs. $480(270,660) \mathrm{min} /$ week, $P=0.026$. A similar relation was found for sports practice-165 $(90,240)$ vs. $135(90,225) \mathrm{min} /$ week, $P=0.017$. Boys and girls had a similar prevalence of fracture history (5.4\% vs. $5.2 \%$, respectively; Table 1$)$.

\section{Body Composition and Fracture}

In a crude analysis, bone properties were not associated with fracture history in either boys or girls (Figure 1). After adjustment for weight, height, and age, girls with fracture history presented decreased subtotal $\mathrm{BMC}(\mathrm{OR}=0.53,95 \%$ CI: $0.32-0.89)$ and aBMD (OR $=0.49,95 \% \mathrm{CI}: 0.28-0.83)$, and decreased LS BMC (OR $=0.69,95 \%$ CI: 0.51-0.93) and aBMD $\quad(\mathrm{OR}=0.67,95 \%$ CI: 0.50-0.92). No significant differences were observed between the non-fracture and fracture groups for subtotal fat mass, lean mass, and fat percentage in either sex.

\section{Physical Activity and Fracture}

We observed an increasing proportion of girls with the history of fracture with increasing time spent in sports practice, whereas a borderline trend was found for time spent in active play (Figure 2). In boys, no associations were found between any measure of physical activity and fracture history.

\section{Physical Activity and BMC/aBMD}

Regarding subtotal measures, we observed that the mean $\mathrm{aBMD}$ and $\mathrm{BMC}$ increased with increasing time spent in sports practice and in active play in both sexes, with the exception of BMC and sports practice in girls (Figure 3). As for LS measures, there were increasing BMC and aBMD trends with increasing time spent in sports practice only in boys.

\section{BMC/aBMD and Fracture by Levels of Physical Activity}

Among girls in the highest category of time spent in active play (over $660 \mathrm{~min} /$ week), history of fracture was associated with decreased subtotal BMC and $\mathrm{aBMD}$, as well as with LS
aBMD-OR $(95 \% \mathrm{CI})=0.27(0.11-0.67), 0.18(0.06-0.49)$, and $0.41(0.22-0.75)$, respectively (Figure 4$)$. When stratified by time spent in sports practice, we observed an association between fracture history and LS BMC and aBMD restricted to girls who practiced more than 225 min of sports per weekOR $\quad(95 \% \quad C I)=0.50 \quad(0.27-0.93)$ and $0.53 \quad(0.28-1.00)$, respectively.

In boys, there were no clear associations between the odds of fracture and subtotal or LS measures in any category of time spent in active play. However, among those in the highest category of sports practice (>240 $\mathrm{min} /$ week), higher subtotal and LS BMC were associated with a lower odds of fracture history-OR $(95 \% \mathrm{CI})=0.39(0.15-0.98)$ and 0.51 $(0.27-0.96)$, respectively.

\section{DISCUSSION}

In this study, we estimated the associations of BMC and aBMD with fracture history by levels of physical activity at 7 years of age in a large subsample of a population-based birth cohort. Our findings show that fractures were likely the result of a biological interaction between $\mathrm{aBMD} / \mathrm{BMC}$ and physical activity: the protective effect of higher aBMD and content on fracture was observed only in children exposed to the highest levels of physical activity. This seems to suggest that the protective role of aBMD and $\mathrm{BMC}$ might require a minimum physical activity threshold, possibly as a proxy for trauma frequency.

We found that boys had higher subtotal aBMD and BMC, whereas girls showed higher LS aBMD. The latter finding is in line with previous studies that showed higher levels of LS bone density in prepubertal girls, most likely due to the osteogenic effect of leptin on trabecular bone. Moreover, boys had higher lean mass, whereas girls had increased fat mass, which is also consistent with previous studies $(27,28)$.

Fracture history had an inverse association with BMC and density, but only among girls and in adjusted estimates. These results generally agree with those obtained in previous studies, where associations are reported even though with modest magnitude. A previous systematic review and meta-analysis 


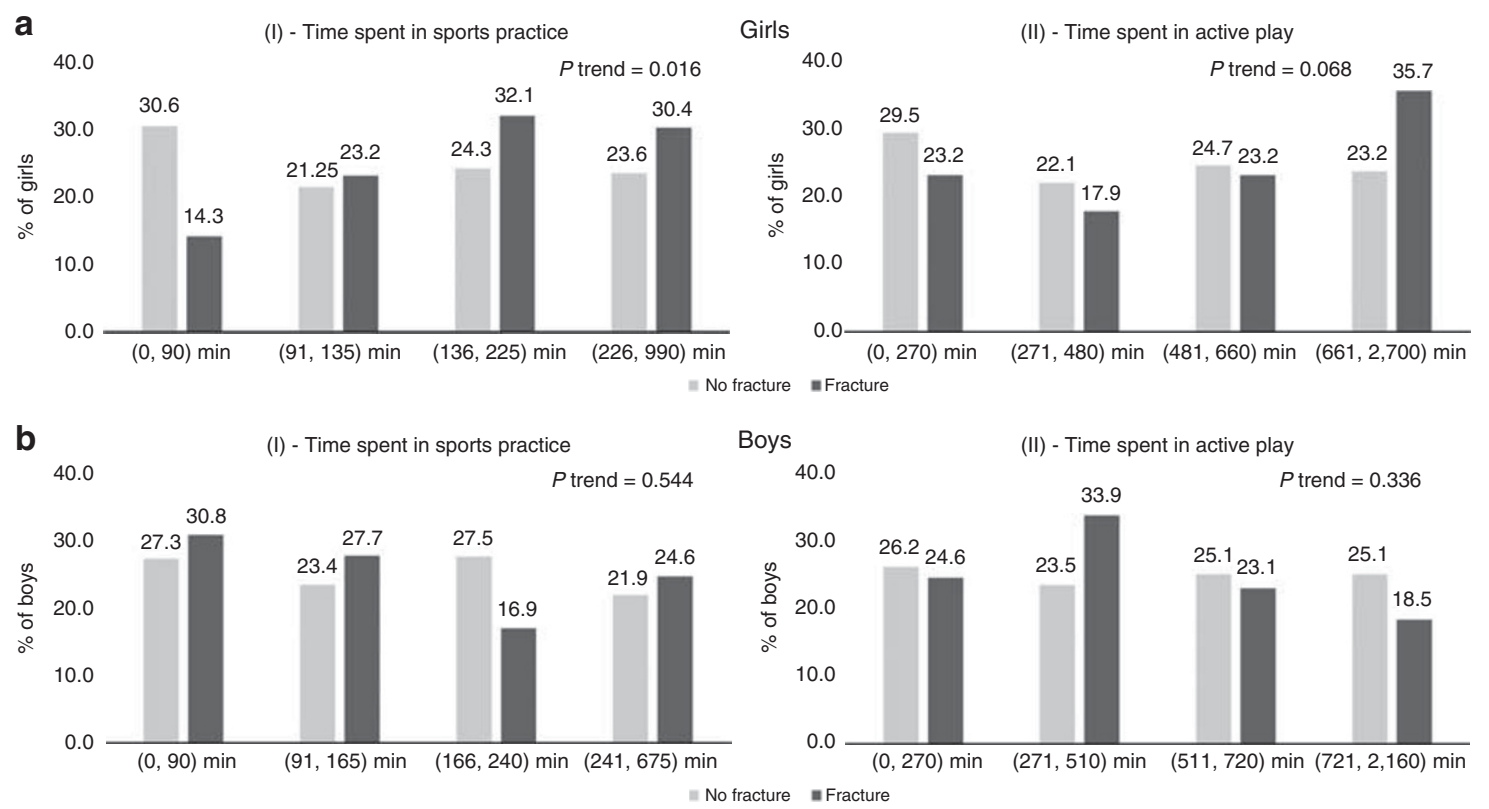

Figure 2. Distribution (\%) of children with and without fracture by categories of weekly time spent in sports practice (I) and time spent in active play (II). (a) Girls; (b) boys.

suggested an association between low bone density and fracture in children aged 0-16 years (ref. 29). A prospective study conducted among 176 healthy boys aged 7 years and followed until 15 years of age also showed that fracture risk during the follow-up was associated with lower aBMD in different locations including femoral neck, total hip, femoral diaphysis, and LS BMD (30). Another recent prospective study of children followed up for a period of 2 years starting at the age of 9.9 years reported that fracture risk was also related to volumetric BMD (6).

As for the role of trauma in our study, fracture history was only associated with regular sports practice and this association was restricted to girls. Although there is some previous evidence of the common notion that children with fractures are more active (31), only one prospective cohort study has demonstrated physical activity as an independent risk factor for fracture (18). A potential explanation for the weak associations found is the notion that physical exertion may have opposing influences on fracture risk, by increasing trauma frequency on the one hand and improving bone physical properties and muscle mass on the other (32). Indeed, studies on the effect of physical activity on bone development consistently report that the practice of physical activity improves bone mass (20-22).

Regarding the positive contribution of physical activity to the bone, we found that boys who spent their most time in organized sports had increased aBMD and BMC. In girls, the associations with subtotal aBMD/BMC were weaker for sports practice but clearer for time spent in active play. This may be the result of a differential response to mechanical stimuli between sexes, or it may reflect that decreased activity levels in girls are not sufficient to reach the necessary threshold; therefore, a positive effect on aBMD and BMC is observed $(33,34)$. An alternative, and possibly more likely, explanation may be the different nature of sports practiced by boys and girls. In our sample, organized activities such as dance and ballet lessons were more frequently reported in girls $(15.7 \%$ vs. $3.5 \%, P<0.001$, and $15.9 \%$ vs. $0.6 \%, P<0.001$, respectively, results not shown), whereas soccer and martial arts were more frequently reported in boys $(22.5 \%$ vs. $0.4 \%$, $P<0.001$, and $14.2 \%$ vs. $7.3 \%, P<0.001$, respectively, results not shown). Differences in the type of sports practiced between sexes may account for the stronger relation of sports with aBMD/BMC in boys, among whom the frequency of trauma-inducing sports is higher than that for girls, and explain the stronger relation of active play with aBMD/BMC in girls, in whom informal activities may be riskier for trauma than organized sports such as dance. This would make organized sports a better proxy for trauma in boys and active play a better proxy in girls.

Regarding the potential role of physical activity as a modifier of the effect of aBMD and BMC on fracture, our results are compatible with a potential interaction according to the levels of physical activity. Associations between aBMD/ BMC and fracture were only clear in children with higher activity levels. As trauma is a necessary cause of fracture, it is possible to speculate that fractures in less active children have resulted from severe, unpredicted trauma, which was not captured through our measurement of regular physical activity. This severe, unpredicted trauma could have caused fracture independently of underlying bone strength. In contrast, in the highest category of physical activity, aBMD and BMC were found to be protective of fracture in both girls and boys, although more consistently in girls. A framework to 
a
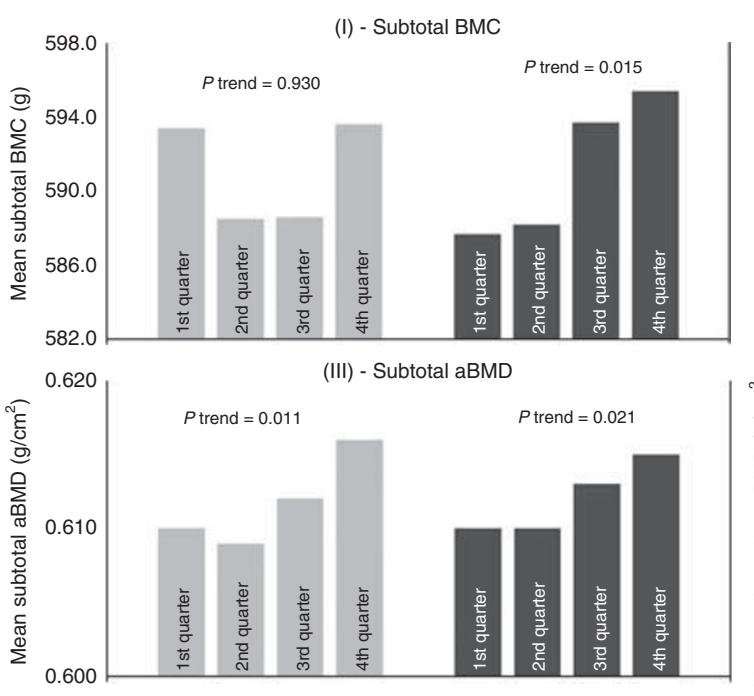

Girls
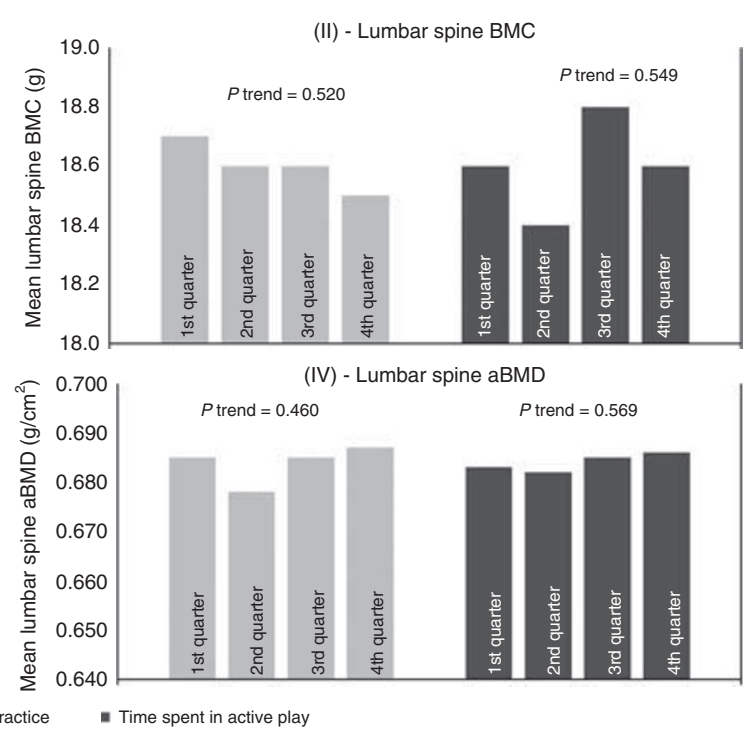

b

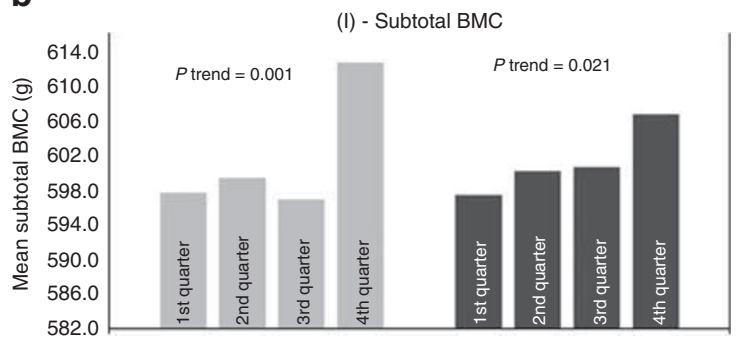

Boys

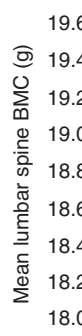

9.6

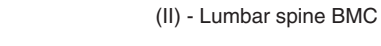

(II) - Lumbar spine BMC
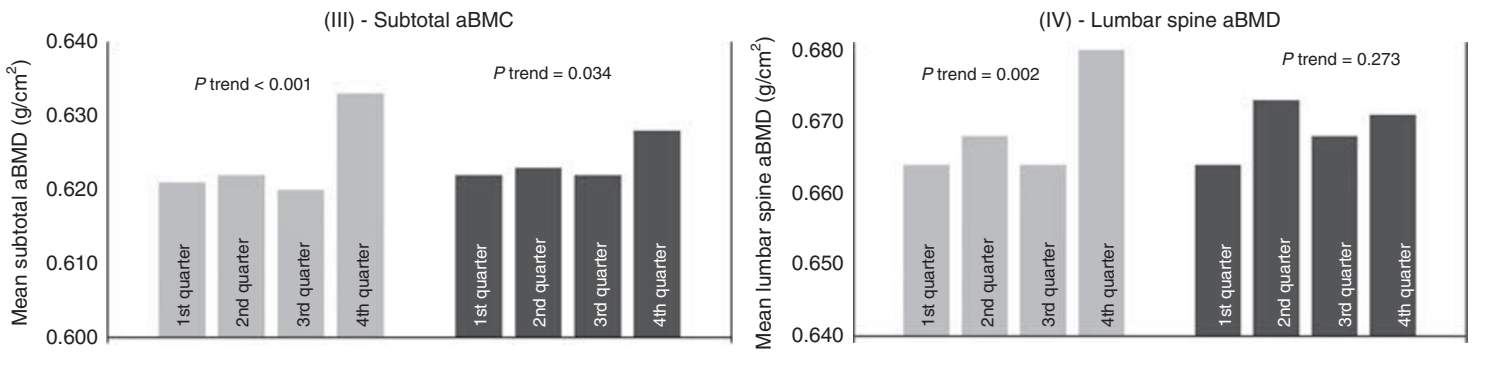

Figure 3. Mean values of bone mineral content (BMC) and areal bone mineral density (aBMD) in each category of time spent in sports practice and time spent in active play per week, adjusted for height, weight, and age. (a) Girls; (b) boys. (I) Subtotal BMC; (II) lumbar spine BMC, (III) subtotal aBMD, (IV) lumbar spine aBMD. In girls, the quarters of time spent in sports practice are [0,90] min, [91, 135] min, [136, 225] min, and [226, 990] min, and for time spent in active play are $[0,270] \mathrm{min},[271,480] \mathrm{min},[481,660] \mathrm{min}$, and $[661,2700] \mathrm{min}$. In boys, the quarters of time spent in sports practice are $[0,90] \mathrm{min},[91,165] \mathrm{min},[166,240] \mathrm{min}$, and $[241,675] \mathrm{min}$, and for time spent in active play are $[0,270] \mathrm{min},[271,510] \mathrm{min},[511$, 720] $\mathrm{min}$, and [721, 2160] $\mathrm{min}$.

interpret our results is the documented threshold effect of physical activity (35), which might require high frequency and/or intensity to uncover a protection of bone quality from fracture risk. In such a case, trauma and lower aBMD/BMC would be synergistic in producing the fracture, but only in children with higher exposure to regular physical activity. An alternative hypothesis would be that the wider range of activity among those children in the highest exertion categories would result in a greater aBMD/BMC range, which would contribute to uncover a relationship between those parameters and fracture. However, further analysis showed very similar ranges of bone parameters between activity categories, regardless of gender, bone measure, or anatomical site (results not shown). Therefore, it seems that the wider range of activity in the highest categories did not result in proportionally wider variations in $\mathrm{aBMD} / \mathrm{BMC}$, and that the heterogeneity in the associations between bone parameters and fracture odds by activity levels was not likely because of different ranges of bone measures.

Until now, only two investigations have studied bone properties, physical activity, and fracture simultaneously, but reached different results. One of those was a prospective 


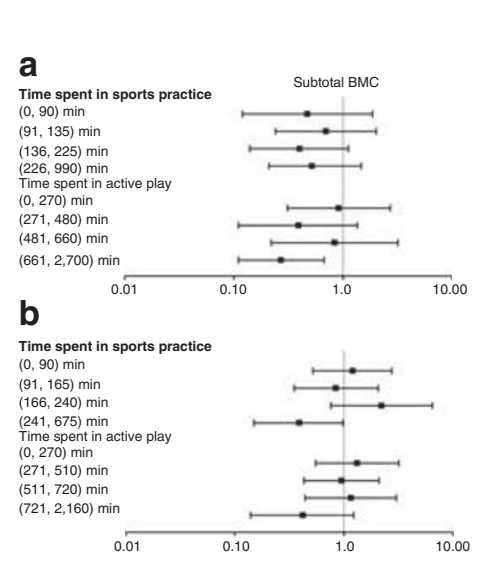

Odds ratio (95\% confidence interval) for fracture per SD increase in bone parameters
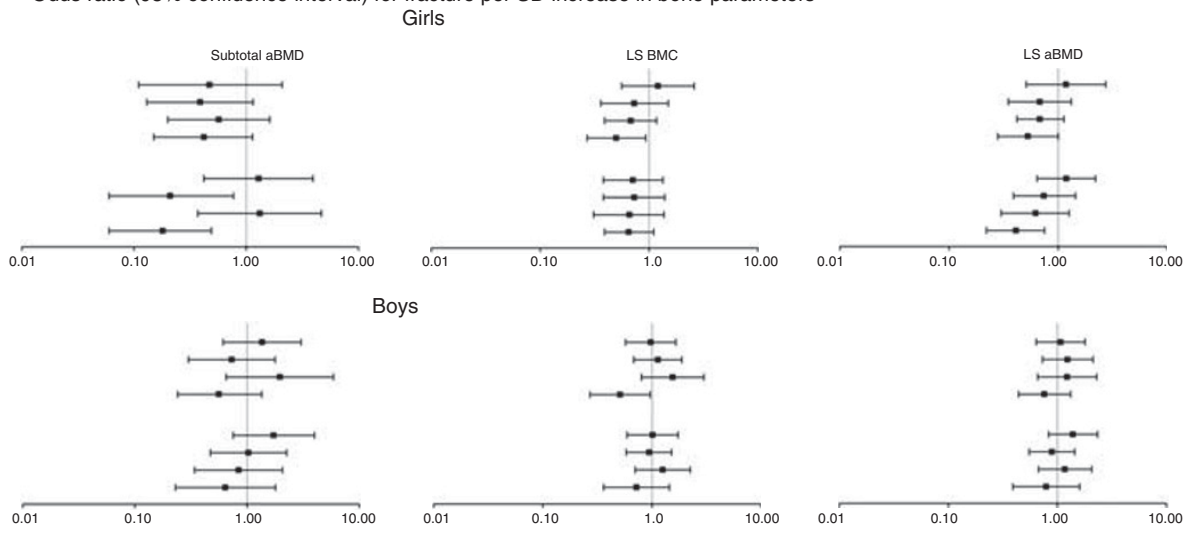

Boys

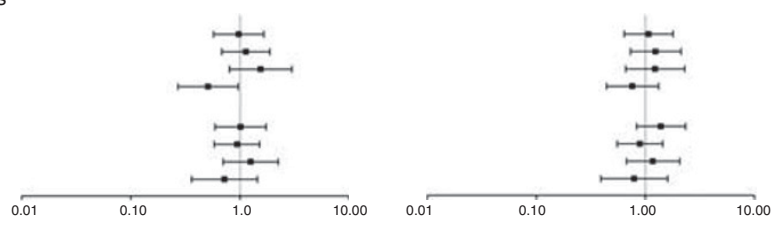

Figure 4. Odds ratios and 95\% confidence intervals for the associations of BMC and aBMD with fracture history stratified by time spent weekly in active play and in sports practice among girls (upper panel, a) and boys (lower panel, b). aBMD, areal bone mineral density; BMC, bone mineral content; $95 \% \mathrm{Cl}$, 95\% confidence interval; LS, lumbar spine. Odds ratios are adjusted for height, weight, and age. The number of children with fracture history was 56 out of 1,055 girls and 65 out of 1,178 boys. From the lowest to the highest level of time spent in active play per week, the numbers of children with fractures were 13, 10,13, and 20 in girls, and 16, 22, 15, and 12 in boys. From the lowest to the highest level of time spent in sports practice per week, the number of children with fractures was $8,13,18$, and 17 in girls, and 20, 15, 14, and 16 in boys.

cohort study that concluded that physical activity was independently associated with fracture (18); the other was a prospective controlled intervention study, which reported that physical activity was associated with decreased fracture risk (19). Diverging results are probably due to the study design, as controlled intervention studies are more likely to optimize the quality of exposure, and therefore its potential benefit, when compared with observational investigations similar to our own.

Some methodological issues should be addressed. First of all, because of the large cohort size, we were not able to confirm fracture history with X-ray or clinical records and relied on data reported by children's parents, which are subject to misreporting. Indeed, Moon et al. (24) showed that $84 \%$ of fractures reported by parents did have radiological confirmation, but the remaining were over-reported, whereas another study in an adult population showed that underreported fractures may underestimate the associations (36). In addition, we assumed that all children were prepubertal at the age of 7 years, which seems plausible based on one of the few nonclinical studies published, where the prevalence of precocious puberty was estimated to be 8 per 10,000 for girls aged 5-9 years and under 1 per 10,000 in boys younger than 8 years in Denmark (37). In addition, intensity of the trauma that caused fracture was not collected, which could have allowed to distinguish mild from moderate and severe trauma. Nonetheless, a prospective study showed that bone fragility contributes to fracture not only in mild but also in moderate and severe trauma (8). In addition, even though DXA is the preferred method to determine bone mass measures due to its speed, precision, low cost, safety, and widespread availability, it has a main disadvantage: DXA does not provide a true volumetric bone mineral density, but an areal BMD. As a consequence, smaller bones are assigned lower aBMD compared with the larger ones (38). Nonetheless, this distortion is less relevant in practice because bones of larger size (diameter) have higher mechanical resistance, independently of volumetric density. This study is limited by its crosssectional design, as aBMD/BMC was measured at 7 years of age, whereas fracture was recorded from 2 years of age. Therefore, it is possible that aBMD/BMC changed between the fracture episode and the DXA scan. However, Foley et al. (11) showed that BMC and aBMD tracked significantly from 8 to 16 years, and Kalkwarf et al. (10) detected tracking from age 6 onward. In addition, although we did not measure tracking of $\mathrm{aBMD} / \mathrm{BMC}$, previous research in this cohort showed substantial tracking of other features of body composition such as fat and fat-free mass from birth up to the age of 7 years (39). Another relevant issue is the possibility of reverse causation, i.e., the possibility that immobilization due to fracture caused decreased aBMD and BMC. Although there is evidence that immobilization after fracture can, in fact, lead to lower aBMD/BMC (40), a small prospective study showed that after the immobilization period, the BMC and aBMD of the fractured arm increased more rapidly than those in the fracture-free arm; therefore, the difference between the arms disappeared after 12 months (41). Moreover, data collected were reported by parents in a face-to-face interview, which is subject not only to memory bias but also to social desirability bias, namely regarding the measurement of physical activity. Finally, we found some background differences between children included in the present subsample when compared with the remaining cohort, but the magnitudes were very small $-0.4 \mathrm{~cm}$ in height, $0.5 \mathrm{~kg}$ in weight, and 0.1 years in age. There were also slight differences in physical activity distribution, which should not be a problem, given that our main analysis was stratified by categories of sports and active play.

The present study has many strengths. First of all, we used data from a population-based birth cohort, which included 


\section{$a B M D / B M C$, physical activity, and fracture}

children born during a short period (2005/2006), thereby avoiding confounding by age or birth cohort. In addition, the study was based on a large sample, which provides more statistical power than most studies conducted in other settings. This is particularly important because previous studies have included children with a wide range of ages (16) or children who are entering the growth spurt $(6,30)$, which features diminished bone strength as a result of faster growth in bone length than width. In this study, we provide evidence that $\mathrm{aBMD}$ and $\mathrm{BMC}$ already contribute to fracture risk prior to the growth spurt, provided children are exposed to enough physical activity. This is consistent with previous studies that found that increased levels of physical activity were beneficial to prepubertal children's bone (42).

Our study revealed that, in a population-based sample of prepubertal children, BMC and density predicted fracture history specifically in the highest levels of physical activity. This is compatible with the hypothesis that higher levels of exertion are necessary to uncover an association between these densitometric bone properties and fracture.

\section{SUPPLEMENTARY MATERIAL}

Supplementary material is linked to the online version of the paper at http://www.nature.com/pr

\section{ACKNOWLEDGMENTS}

We gratefully acknowledge the families enrolled in the Generation XXI for their kindness, all members of the research team for their enthusiasm and perseverance, and the participating hospitals and their staff for their help and support.

\section{STATEMENT OF FINANCIAL SUPPORT}

Generation XXI was funded by FEDER through the Operational Programme Competitiveness and Internationalization and national funding from the Foundation for Science and Technology-FCT (Portuguese Ministry of Science, Technology and Higher Education; POCI-01-0145-FEDER-016838 and POCI-01-0145-FEDER-016837), under the projects "BioAdversity: Como a adversidade social na infância condiciona a saúde: A biologia da adversidade social" (Ref. FCT PTDC/DTP-EPI/1687/2014) and "PathMOB.: Risco cardiometabólico na infância: desde o início da vida ao fim da infância" (Ref. FCT PTDC/DTP-EPI/3306/2014); by the Unidade de Investigação em Epidemiologia-Instituto de Saúde Pública da Universidade do Porto (EPIUnit; POCI-01-0145-FEDER-006862; Ref. UID/DTP/ 04750/2013); and by Administração Regional de Saúde Norte (Regional Department of Ministry of Health) and Fundação Calouste Gulbenkian; PhD grant SFRH/BD/92370/2013 (T.M.) and postdoc grant SFRH/BPD/ 88729/2012 (R.L.), co-funded by FCT and the POPH/FSE Program; and an MSD merit scholarship attributed to the Masters in Public Health of Universidade do Porto (A.M.). This study is also a result of the project DOCnet (NORTE-01-0145-FEDER-000003), supported by Norte Portugal Regional Operational Programme (NORTE 2020), under the PORTUGAL 2020 Partnership Agreement, through the European Regional Development Fund (ERDF).

Disclosure: The authors declare no conflict of interest.

\section{REFERENCES}

1. Rennie L, Court-Brown CM, Mok JY, Beattie TF. The epidemiology of fractures in children. Injury 2007;38:913-22.

2. Landin LA. Epidemiology of children's fractures. J Pediatr Orthop B 1997;6:79-83.
3. Cooper C, Dennison EM, Leufkens HG, Bishop N, van Staa TP. Epidemiology of childhood fractures in britain: a study using the general practice research database. J Bone Miner Res 2004;19:1976-81.

4. Mäyränpää $M K$, Mäkitie $O$, Kallio $P E$. Decreasing incidence and changing pattern of childhood fractures: a population-based study. J Bone Miner Res 2010;25:2752-9.

5. Clark EM. The epidemiology of fractures in otherwise healthy children. Curr Osteoporos Rep 2014;12:272-8.

6. Clark EM, Ness AR, Bishop NJ, Tobias JH. Association between bone mass and fractures in children: a prospective cohort study. J Bone Miner Res 2006;21:1489-95.

7. Farr JN, Amin S, Melton LJ 3rd, et al. Bone strength and structural deficits in children and adolescents with a distal forearm fracture resulting from mild trauma. J Bone Miner Res 2014;29:590-9.

8. Clark EM, Ness AR, Tobias JH. Bone fragility contributes to the risk of fracture in children, even after moderate and severe trauma. J Bone Miner Res 2008;23:173-9.

9. Ben-Shlomo Y, Kuh D. A life course approach to chronic disease epidemiology: conceptual models, empirical challenges and interdisciplinary perspectives. Int J Epidemiol 2002;31:285-93.

10. Kalkwarf HJ, Gilsanz V, Lappe JM, et al. Tracking of bone mass and density during childhood and adolescence. J Clin Endocrinol Metab 2010;95:1690-8.

11. Foley S, Quinn S, Jones G. Tracking of bone mass from childhood to adolescence and factors that predict deviation from tracking. Bone 2009;44:752-7.

12. Farr JN, Khosla S, Achenbach SJ, et al. Diminished bone strength is observed in adult women and men who sustained a mild trauma distal forearm fracture during childhood. J Bone Miner Res 2014;29:2193-02.

13. Buttazzoni C, Rosengren BE, Tveit M, Landin L, Nilsson JA, Karlsson MK. Does a childhood fracture predict low bone mass in young adulthood? A 27-year prospective controlled study. J Bone Miner Res 2013;28:351-9.

14. Marshall D, Johnell O, Wedel H. Meta-analysis of how well measures of bone mineral density predict occurrence of osteoporotic fractures. Br Med J 1996;312:1254-9.

15. Dimitri P, Wales JK, Bishop N. Fat and bone in children: differential effects of obesity on bone size and mass according to fracture history. J Bone Miner Res 2010;25:527-36.

16. Goulding A, Grant AM, Williams SM. Bone and body composition of children and adolescents with repeated forearm fractures. J Bone Miner Res 2005;20:2090-6.

17. Nauta J, Martin-Diener E, Martin BW, van Mechelen W, Verhagen E. Injury risk during different physical activity behaviours in children: a systematic review with bias assessment. Sports Med 2015;45:327-36.

18. Clark EM, Ness AR, Tobias JH. Vigorous physical activity increases fracture risk in children irrespective of bone mass: a prospective study of the independent risk factors for fractures in healthy children. J Bone Miner Res 2008;23:1012-22.

19. Fritz J, Coster ME, Nilsson JA, Rosengren BE, Dencker M, Karlsson MK. The associations of physical activity with fracture risk-a 7-year prospective controlled intervention study in 3534 children. Osteoporos Int 2015;27:915-22.

20. Behringer M, Gruetzner S, McCourt M, Mester J. Effects of weightbearing activities on bone mineral content and density in children and adolescents: a meta-analysis. J Bone Miner Res 2014;29:467-78.

21. Janz KF, Letuchy EM, Burns TL, Eichenberger Gilmore JM, Torner JC, Levy SM. Objectively measured physical activity trajectories predict adolescent bone strength: Iowa bone development study. Br J Sports Med 2014;48:1032-6.

22. Harvey NC, Cole ZA, Crozier SR, et al. Physical activity, calcium intake and childhood bone mineral: a population-based cross-sectional study. Osteoporos Int 2012;23:121-30.

23. Alves E, Lunet N, Correia S, Morais V, Azevedo A, Barros H. Medical record review to recover missing data in a portuguese birth cohort: agreement with self-reported data collected by questionnaire and inter-rater variability. Gac Sanit 2011;25:211-9. 


\section{Articles | Martins et al.}

24. Moon RJ, Lim A, Farmer M, et al. Validity of parental recall of children's fracture: implications for investigation of childhood osteoporosis. Osteoporos Int 2016;27:809-13.

25. Fassier A, Gaucherand P, Kohler R. Fractures in children younger than 18 months. Orthop Traumatol Surg Res 2013;99:S160-70.

26. Wegmann H, Orendi I, Singer G, et al. The epidemiology of fractures in infants - which accidents are preventable? Injury 2016;47:188-91.

27. Garnett SP, Hogler W, Blades B, et al. Relation between hormones and body composition, including bone, in prepubertal children. Am J Clin Nutr 2004;80:966-72.

28. Arabi A, Tamim H, Nabulsi M, et al. Sex differences in the effect of bodycomposition variables on bone mass in healthy children and adolescents. Am J Clin Nutr 2004;80:1428-35.

29. Clark EM, Tobias JH, Ness AR. Association between bone density and fractures in children: a systematic review and meta-analysis. Pediatrics 2006;117:e291-7.

30. Chevalley T, Bonjour JP, van Rietbergen B, Ferrari S, Rizzoli R. Fractures during childhood and adolescence in healthy boys: relation with bone mass, microstructure, and strength. J Clin Endocrinol Metab 2011;96: 3134-42.

31. Ma D, Jones G. Television, computer, and video viewing; physical activity; and upper limb fracture risk in children: a population-based case control study. J Bone Miner Res 2003;18:1970-7.

32. Bailey DA, Faulkner RA, McKay HA. Growth, physical activity, and bone mineral acquisition. Exerc Sport Sci Rev 1996;24:233-66.

33. Kriemler S, Zahner L, Puder JJ, et al. Weight-bearing bones are more sensitive to physical exercise in boys than in girls during preand early puberty: a cross-sectional study. Osteoporos Int 2008;19: 1749-58.
34. Cardadeiro G, Baptista F, Ornelas R, Janz KF, Sardinha LB. Sex specific association of physical activity on proximal femur bmd in 9 to 10 year-old children. PLoS ONE 2012;7:e50657.

35. Bachrach LK. Acquisition of optimal bone mass in childhood and adolescence. Trends Endocrinol Metab 2001;12:22-8.

36. Siggeirsdottir K, Aspelund T, Sigurdsson G, et al. Inaccuracy in self-report of fractures may underestimate association with health outcomes when compared with medical record based fracture registry. Eur J Epidemiol 2007;22:631-9.

37. Teilmann G, Pedersen CB, Jensen TK, Skakkebaek NE, Juul A. Prevalence and incidence of precocious pubertal development in Denmark: an epidemiologic study based on national registries. Pediatrics 2005;116:1323-8.

38. Crabtree NJ, Arabi A, Bachrach LK, et al. Dual-energy x-ray absorptiometry interpretation and reporting in children and adolescents: the revised 2013 iscd pediatric official positions. J Clin Densitom 2014;17:225-42.

39. Fonseca MJ, Severo M, Correia S, Santos AC. Effect of birth weight and weight change during the first $96 \mathrm{~h}$ of life on childhood body composition-path analysis. Int J Obes 2015;39:579-85.

40. Ceroni D, Martin X, Delhumeau C, Rizzoli R, Kaelin A, Farpour-Lambert $\mathrm{N}$. Effects of cast-mediated immobilization on bone mineral mass at various sites in adolescents with lower-extremity fracture. J Bone Joint Surg Am 2012;94:208-16.

41. Fung EB, Humphrey ML, Gildengorin G, Goldstein N, Hoffinger SA. Rapid re-mineralization of the distal radius following forearm fracture in children. J Pediatr Orthop 2011;31:138-43.

42. Farr JN, Blew RM, Lee VR, Lohman TG, Going SB. Associations of physical activity duration, frequency, and load with volumetric BMD, geometry, and bone strength in young girls. Osteoporos Int 2011;22: 1419-30. 